Cahiers $d u$ MONDE RUSSE

\section{Cahiers du monde russe}

Russie - Empire russe - Union soviétique et États indépendants

$50 / 4 \mid 2009$

Varia

\title{
L'utopie décembriste de A. Ulybyšev « Un rêve » (1819)
}

Publication de l'original en français

The A.D. Ulybyshev's Decembrist utopia "A Dream" (1819). Publication of the French original manuscript

\section{Michel Niqueux}

\section{OpenEdition Journals}

Édition électronique

URL : https://journals.openedition.org/monderusse/9916

DOI : $10.4000 /$ monderusse. 9916

ISSN : $1777-5388$

Éditeur

Éditions de l'EHESS

Édition imprimée

Date de publication : 15 décembre 2009

Pagination : 737-755

ISBN : 978-2-7132-2261-0

ISSN : $1252-6576$

\section{Référence électronique}

Michel Niqueux, "L'utopie décembriste de A. Ulybyšev « Un rêve » (1819) », Cahiers du monde russe [En ligne], 50/4 | 2009, mis en ligne le 14 janvier 2013, consulté le 04 septembre 2022. URL : http:// journals.openedition.org/monderusse/9916; DOI : https://doi.org/10.4000/monderusse.9916 


\title{
L'UTOPIE DÉCEMBRISTE DE A. ULYBYŠEV «UN RÊVE» (1819)
}

\author{
Publication de l'original en français
}

La petite utopie d'Aleksandr Ulybyšev, «Un rêve » (1819), publiée en 1927 est bien connue : elle figure dans les anthologies de textes des décembristes, d'utopies ou de récits fantastiques russes², elle est mentionnée dans les histoires de l'utopie ${ }^{3}$, elle a fait l'objet d'un article de M.V. Nečkina ${ }^{4}$, elle est traduite en

1. « $\mathrm{K}$ istorii "Zelënoj lampy" » [«Contribution à l'histoire de "La Lampe verte" »], in B. Modzalevskij, Dekabristy i ih vremja [Les décembristes et leur temps], M. : Izd. Ob-va političeskih katoržan, t. 1, 1927, p. 53-56.

2. I.Ja. Ščipanov, éd., Izbrannye social'no-političeskie i filosofskie proizvedenija dekabristov [Anthologie de textes socio-politiques et philosophiques des décembristes], M. : Gospolitizdat, t. I, 1951, p. 286-292 ; V. Orlov, éd., Dekabristy : Poèzija, dramaturgija, proza, publicistika, literaturnaja kritika [Les décembristes : Poésie, théâtre, prose, écrits politiques et sociaux, critique littéraire], M.-L. : GIXL, 1951, p. 563-566; V. Guminskij, éd., Vzgljad skvoz' stoletija: Russkaja fantastika XVIII i načala XIX VV. [Regard à travers les siècles : La littérature fantastique russe des XVIII -début du XIX ${ }^{\mathrm{e}}$ siècle], M. : Molodaja gvardija, 1977, p. 93-99 ; S.S. Volk, éd., «Ih večen s vol'nost’ju sojuz » : Literaturnaja kritika i publicistika dekabristov ["Éternelle est leur alliance avec la liberté »: La critique littéraire, les écrits politiques et sociaux des décembristes], M. : Sovremennik, 1983, p. 226-231 ; V.P. Šestakov, éd., Russkaja literaturnaja utopija [L'utopie littéraire russe], M.: Izd-vo Moskovskogo Universiteta, 1986, p. 87-91 ; Inga N. Fomina, éd., Sil'fida : Fantastičeskie povesti russkih romantikov [Sylphide : Nouvelles fantastiques des romantiques russes], M. : Sovremennik, 1988, p. 216-222 ; A.A.Zlatopol'skaja, éd., Ž.-Ž. Russo : pro et contra [J.-J. Rousseau : pro et contra], SPb. : RXGI, 2005, p. 170-175.

3. L. Heller, M. Niqueux, Histoire de l'utopie en Russie. P. : PUF (Écriture), 1995, p. 114-115 ; traduction allemande : Geschichte der Utopie in Russland, Bietigheim-Bissingen : Ed. Tertium, 2003 ; traduction russe : L. Geller, M. Nikë, Utopija v Rossii, SPb. : Giperion, 2003 ; B.F. Egorov, Rossijskie utopii : Istoričeskij putevoditel' [Les utopies russes : Guide historique], $\mathrm{SPb}$. : Iskusstvo-SPB, 2007, p. 124-125.

4. M.V. Nečkina, « Dekabristskaja "Utopija”. K analizu političeskoj programmy Sojuza blagodentsvija » [ «Une "Utopie" décembriste. Contribution à l'analyse du programme politique de l'Union du bien public »], in Iz istorii social'no-političeskih idej (K 70-letiju ak. V.P. Volgina) [Histoire des idées socio-politiques. Pour le soixante-dixième anniversaire de l'académicien V.P. Volgin], M. : AN SSSR, 1955, p. 376-384. 
italien ${ }^{5}$. Mais elle n'était connue jusqu'ici qu'en traduction russe ${ }^{6}$, l'original en français restant inédit et sans référence précise. Le manuscrit se trouve dans les «Puschkiniana » («Papiers de la société Zelënaja lampa ») des archives de la Maison Pouchkine, à Saint-Pétersbourg (f. 244, op. 36, n 32), avec deux autres textes en français de 1819 - un tableau satirique des sociétés conservatrices et occidentalisées de Saint-Pétersbourg ${ }^{7}$, et une «Conversation entre Bonaparte et un voyageur anglais »- que nous nous apprêtons à publier par ailleurs ${ }^{8}$. Ces manuscrits sont anonymes, mais le grand pouchkiniste B.L. Modzalevskij (1874-1928), qui les a découverts dans les archives de Russkaja starina, a pu les attribuer à Ulybyšev à la suite d'une analyse graphologique 9 .

Ulybyšev était membre de La Lampe verte (Zelënaja lampa), un cénacle littéraire (mentionné dans «Un rêve »), qui servit en 1818-1820 de filiale (pobočnaja uprava), ou de paravent à l' «Union du bien public » (Sojuz blagodenstvija ${ }^{10}$ ), comme l'ont établi Modzalevskij (à la suite de P. Ščegolev) puis Nečkina, sans que ses membres, pour la plupart, fussent au courant de cette filiation. Les réunions de La Lampe verte n'étaient cependant pas publiques, et ses membres, au nombre de 21 , faisaient le serment de garder le secret ${ }^{11}$. Ulybyšev, qui appartenait aux deux organisations, connaissait sans doute leurs liens de dépendance, mais pas Puškin, qui fréquentait seulement La Lampe verte ${ }^{12}$. «Un rêve » fut lu

5. «Un sogno », in Utopisti russi del primo ottocento (a cura di Marina Rossi Varese), Naples : Guida editori, 1982, p. 45-53 (et p. 11-17 de l'introduction).

6. Traduction (excellente) de V.B. Vraskaja-Jančevskaja, collaboratrice du Puškinskij dom. Notons la traduction de mystérieux par mističeskoe, de ruban par orden, de magistrat suprême par verhovnyj činovnik.

7. « Lettre à un ami d'Allemagne sur les sociétés de Pétersbourg » (1819).

8. Respectivement dans la Revue des études slaves et dans la revue en ligne Napoleonica.

9. Modzalevskij, «K istorii “Zelënoj lampy” », p. 41-44.

10. Blagodenstvie peut être aussi traduit par prospérité. Herzen dit en français l' « Alliance du bien-être » [Études historiques sur les héros de 1825 et leurs prédécesseurs, d'après leurs mémoires (1868, en français)], Sobranie sočinenij v 30 tt. [Euvres en 30 vol.], t. XX, M. : 1960, p. 191. Sur le lien entre Sojuz blagodentsvija et Zelënaja lampa, voir la dénonciation de M.K. Gribovskij présentée par A. Benckendorf à Alexandre I ${ }^{\mathrm{er}}$ Dekabristy : Otryvki iz istočnikov [Les décembristes. Extraits des sources], in Ju.G. Oksman, éd., M.-L. : GIZ, 1926, p. 111. En français, on trouvera un exposé sur l'Union du bien public dans la thèse de Julie Morel-Grandhaye, La République des décembristes. Pour une histoire de la modernité politique en Russie (1760-1870), dir. Marie-Pierre Rey, Paris-I, 2008, vol. I, p. 349-381 ; mais l'utopie d'Ulybyšev n'est pas mentionnée.

11. Les membres les plus connus de La Lampe verte sont : A.S. Puškin, N.V. Vsevoložskij, Jakov Tolstoj, A.A. Del'vig, S.P. Trubeckoj, P.P. Kaverin, Fedor Glinka, A. Jakubovič (cf. V.M. Bokova, Èpoha tajnyh obščestv : Russkie obščestvennye ob"edinenija pervoj treti XIX V [L'époque des sociétés secrètes. Les sociétés du premier tiers $d u X I X^{\mathrm{e}} \mathrm{s}$.], M. : Realii Press, 2003, p. 225. L'auteur rappelle la réputation orgiaque de La Lampe verte, mais sans distinguer les « réunions de travail », dont l'ordre du jour a été conservé, des soirées bien arrosées qui avaient lieu le samedi chez N.V. Vsevoložskij (cf. B. Tomaševskij, Puškin, kn. 1-aja, 18131824 [Puškin, livre I, 1813-1824], M. -L. : Izd. AN SSSR, 1956, p. 205).

12. Cf. « Goriš li ty, lampada naša... » (Iz pis’ma k Ja.N. Tolstomu ) [« Brûles-tu, lampe nôtre... », extrait d'une lettre à Ja.N. Tolstoj]. Puškin et Ulybyšev se rencontraient certainement à La Lampe verte (cf. L.A. Čerejskij, Puškin i ego okruženie [Puškin et son entourage], 
par Ulybyšev à la $13^{\mathrm{e}}$ séance de La Lampe verte, qui se tint à la fin de l'année 1819 chez N.V. Vsevoložskij, collègue (au Collège des Affaires étrangères) et ami de Puškin, fondateur de La Lampe verte. Ulybyšev, mentionné par S.P. Trubeckoj parmi les membres de La Lampe verte (mais non de l'Union du bien public), ne fut pas inculpé par la Commission d'enquête, qui ne considéra pas La Lampe verte comme une organisation poursuivant des buts politiques. Du reste, il n'avait pas suivi la radicalisation du mouvement décembriste.

Ulybyšev ne figure pas dans les Histoires de la littérature ni dans les encyclopédies littéraires. En revanche, il est mentionné dans les Histoires de la musique russe, car il fut un critique musical et un musicologue de renommée européenne, comme on le verra plus loin. C'est l' « aperçu biographique » publié en 1886 dans Russkij $\operatorname{arhiv}\left(\mathrm{n}^{\circ} 1\right)$ par A.S. Gaciskij ${ }^{13}$ qui est à ce jour la principale source de renseignements sur la vie et l'œuvre d'Aleksandr Dmitrievič Ulybyšev (1794-1858).

Ulybyšev est né à Dresde ${ }^{14}$ le 2 avril 1794. Selon une légende familiale, il descendrait de la fille du prince Dmitrij Donskoj, Ulyba. Il vécut jusqu'à l'âge de seize ans en Saxe, où son père était ambassadeur. À son arrivée en Russie, Ulybyšev passa

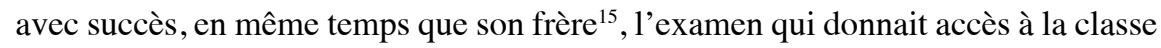
inférieure des fonctionnaires. En août 1812, il commença sa carrière au secrétariat du ministre des Finances, puis passa aux Mines et Salines (31 août 1813 29 février 1816) et enfin, en avril 1816, au Collège des Affaires étrangères ${ }^{16}$ (auquel en 1817-1820 Puškin était également attaché), d'abord à Saint-Pétersbourg comme traducteur du russe en français et inversement (avec le $9^{\mathrm{e}}$ grade en 1817), puis en octobre 1817 au cabinet du chargé d'affaires du ministère à Moscou, où il monta régulièrement en grade ( $8^{\mathrm{e}}$ grade en $1820,7^{\mathrm{e}}$ en $1824,6^{\mathrm{e}}$ en $1825,5^{\mathrm{e}}$ en 1828$)$ et fut souvent décoré et récompensé par le souverain ${ }^{17}$. De 1825 à 1829 , il collabore au Journal de Saint-Pétersbourg, organe en français du ministère des Affaires

L. : Nauka, 1988, p. 454 ; B. Tomaševskij, op. cit., p. 207 ; Puškinskaja Ènciklopedija 17991999 [Encyclopédie Puškin 1799-1999], M. : Ast, 1999, p. 575-576).

13. A.S. Gaciskij, « A.D. Ulybyšev. Biografičeskij očerk » (A.D. Ulybyšev. Aperçu biographique), Russkij arhiv 1, 1886, p. 55-68 (disponible à l'adresse Internet suivante : http://bibliotekar.ru/reprint-63/index.htm). A.S. Gaciskij (1838-1893) : statisticien, archiviste, ethnographe de la région de Nižnij Novgorod (cf. l'encyclopédie Brockhaus et Efron, où son nom est orthographié avec deux $s$ ).

14. Selon François-Jospeh Fétis, compositeur, critique musical et musicographe belge (17841871), qui connaissait Ulybyšev et lui consacra une notice (qui contient de nombreuses erreurs) dans sa Biographie universelle des musiciens (2e éd., P. : F. Didot, 1864, t. 6, p. 390). Gaciskij ignorait le lieu de naissance d'Ulybyšev.

15. Vladimir Dmitrievič, par la suite professeur aux Ponts et chaussées et membre du comité d'organisation de la cathédrale Saint-Isaac.

16. L'oukase du 8/20 septembre 1802 portant création des ministères fut suivi le même jour d'un autre oukase maintenant l'existence du Collège des Affaires étrangères, qui passa simplement sous la direction du ministre des Affaires étrangères, et ne fut supprimé qu'en 1832 (oukase du 30 avril) ; cf. http://www.montreal.mid.ru/dip_11.html

17. Gaciskij, « A.D. Ulybyšev », p. 57, d'après les états de service d'Ulybyšev. 
étrangères ${ }^{18}$. En 1829 , il fut pressenti pour succéder à Griboedov en Perse, mais refusa ce poste. À la mort de son père, en 1830, il prit sa retraite, avec le grade de conseiller d'État actif, c'est-à-dire le quatrième rang, et s'installa dans la propriété paternelle dont il avait hérité, à Lukino, non loin de Nižnij Novgorod, où à partir de 1840 il passe tous les hivers et s'adonne à ses deux passions artistiques, la musique et le théâtre. La musique étant un motif important d' " Un rêve », nous parlerons du musicologue à la suite du texte de l'utopie. Ulybyšev meurt le 29 janvier 1858 à Nižnij Novgorod.

L'utopie d'Ulybyšev se situe dans la tradition du Staatsroman du XVIII ${ }^{\mathrm{e}}$ siècle, avec la description d'un État et d'un gouvernement parfaits. Ulybyšev, qui se déclare « patriote », « ami de la raison » et « philanthrope » ${ }^{19}$, témoigne d'un décembrisme modéré, constitutionnel-monarchiste plutôt que républicain (ce n'est qu'en 1820 que l'Union du bien public se prononcera pour le républicanisme, à la suite d'un exposé de Pestel $^{20}$ ). La société découverte en rêve par Ulybyšev est une société de citoyens libres et égaux devant la loi, représentés par des « anciens » qui sont tour à tour magistrats et maîtres des cérémonies religieuses. Ulybyšev laisse dans l'ombre certains aspects de sa société utopienne, économiques notamment, pour en développer trois principaux, la réalité (militaire) ayant réveillé le rêveur : la religion (l'orthodoxie, la «confession gréco-catholique », a été remplacée par une sorte de culte de l'Être suprême, sans prêtres ni moines ${ }^{21}$ ), l'armée (l'armée de métier a été remplacée par une armée de réservistes, les casernes ont été transformées en écoles et en bibliothèques), le rapport à l'Occident, avec une critique de la précipitation de Pierre le Grand à civiliser son peuple, qui a retardé l'éclosion du génie national. Comme dans sa «Lettre à un ami d'Allemagne sur les sociétés de Pétersbourg », Ulybyšev préconise une assimilation critique de la civilisation occidentale et, pour la littérature, un recours à la «veine féconde et presque intacte de nos antiquités et de nos traditions populaires ». Bien qu'éduqué à l'étranger, Ulybyšev est un préslavophile modéré, un « patriote », comme se définissaient les membres de l'Union du bien public.

18. Contrairement à ce qu'écrit Gaciskij, Ulybyšev n'était pas rédacteur en chef de ce journal, mais un de ses correspondants, de 1825 à 1829 : cf. N. Speranskaja, « Peterburgskaja gazeta Le Furet/Le Miroir (1829-1833) », Novoe literaturnoe obozrenie 94, 2008 (http://magazines.russ. $\mathrm{ru} / \mathrm{nlo} / 2008 / 94 / \mathrm{sp} . h t m l$ [note 4]). La Grande Encyclopédie soviétique indique aussi qu'il fut de 1816 à 1824 rédacteur du Conservateur impartial (fondé en 1813 par l'abbé Manguin, émigré, précepteur de S.S. Uvarov).

19. La philanthropie (ou humanitarisme, qui remplace le terme de philanthropie dans les années 1830), čelovekoljubie, était l'un des quatre domaines d'activité de l'Union du bien public, avec l'enseignement, la justice et l'économie (cf. les statuts de l'Union in Izbrannye social'no-političeskie i filosofskie proizvedenija dekabristov, t. I, et : http://decemb.hobby.ru/ index.shtml?archive/blago).

20. Nečkina, « Dekabristskaja “Utopija”.... », p. 382.

21. Ulybyšev est ici plus dans l'esprit des Lumières que Pestel', qui dans son projet de constitution (Russkaja Pravda, Le code russe), stipulait que « la religion (vera) gréco-russienne doit être reconnue comme la religion dominante du grand État russien » (Izbrannye social'nopolitičeskie i filosofskie proizvedenija dekabristov, t. I, p. 157). 
Tout en ne présentant pas un tableau complet de l'organisation de la polis russe en l'an 2119, l'utopie d'Ulybyšev n'est pas à classer dans les « œuvres littéraires » lues aux séances de la Lampe verte : c'est au contraire le seul texte politique qui y fut lu, et nous sommes enclin à y voir l'exposé du programme politique de l'Union du bien-être, qui, on le sait, n'a pas été rédigé du fait des divergences entre modérés et radicaux (Pestel') qui aboutiront à la création de la Société du sud (1821) et à celle du Nord (1822) de N. Murav'ev, plus modérée. Ulybyšev se fait le porteparole des « désirs et [des] vœux unanimes de [ses] respectables collègues ». «Un rêve » joue en quelque sorte le rôle de seconde partie des statuts de l'Union et doit être à ce titre placé sur le même plan que les projets de constitution de Murav'ev et de Pestel'. C'est dire son importance.

Le recours à la fiction du rêve pour donner une vision du futur avait déjà une tradition dans la littérature utopique russe : «Un rêve. Une société heureuse » (1759) de Sumarokov, vision d'une société sans classes mue par l'idéal du bien public, basée sur une piété « sans mélange de superstition » et sur la loi naturelle, le chapitre « Spasskaja Polest'» du Voyage de Pétersbourg à Moscou (1790) de Radiščev (qui démasque l'image du souverain idéal que voulait incarner Catherine II) ; en France, le roman de Louis-Sébastien Mercier L'an deux mille quatre cent quarante, rêve s'il en fut jamais (1771), dont on trouve peut-être un écho dans le temple que décrit Ulybyšev $^{22}$. Comme chez Mercier, il n'y a pas u-topie au sens étymologique du mot, puisque c'est une anticipation, un voyage dans le temps et non dans l'espace,

22. Il y a aussi chez Mercier une vision de la Russie en l'an 2440, accompagnée d'un hommage à Catherine II qui est bien dans l'esprit du temps : «La nation russe est considérablement à la mode dans ce moment-ci [1782] à la ville et à la cour », rapporte le baron de Grimm (A. Lortholary, Les «philosophes » du XVIII ${ }^{e}$ siècle et la Russie : Le mirage russe en France au XVIII ${ }^{e}$ siècle. P. : Boivin et $C^{\text {ie }}, 1951$, p. 167). Le chapitre 42 (ou 48 selon les éditions) contient des extraits de « gazettes » du monde entier (de l'an 2440), dont une correspondance de Pétersbourg :

«De Pétersbourg, le...

«Le plus beau de tous les titres est celui de Législateur. Un souverain est presque un dieu pour une nation lorsqu'il lui donne des loix sages \& constantes. On répete encore avec transport le nom de l'auguste Catherine II : on ne s'entretient plus de ses conquêtes \& de ses triomphes ; on parle de ses loix. Son ambition fut de dissiper les ténebres de l'ignorance, de substituer à des coutumes barbares des loix dictées par l'humanité. Plus heureuse, plus grande que Pierre le Grand, parce qu'elle fut plus humaine, elle s'appliqua, malgré tant d'exemples contraires, à faire de son peuple un peuple heureux \& florissant. Il le fut, malgré les orages publics \& domestiques qui battirent son trône \& l'ébranlerent. Son courage a su raffermir une couronne que l'univers se plaisoit à voir sur son front. Il faut remonter dans l'antiquité la plus reculée, pour rencontrer un législateur qui ait eu autant de dignité \& de profondeur. - Les fers qui chargeoient le laboureur ont été brisés : il a levé la tête \& s'est vu avec joie au rang des hommes. L'artisan du luxe a cessé de voir sa profession plus lucrative \& plus honorable. Le génie de l'humanité a dit à tout le nord: Hommes! Soyez libres; \& souvenez-vous, races futures, que c'est à une femme que vous devez ce que vous êtes. »

«Selon le dernier dénombrement des habitans de toutes les Russies, le relevé monte à quarante-cinq millions d'hommes. On n'en comptoit que quatorze en 1769. Mais la sagesse du législateur, son code humain, le trône de ses successeurs solidement affermi, parce qu'ils furent généreux \& populaires, tout a rendu la population égale à l'étendue de cet empire, plus vaste que celui des Romains, que celui d'Alexandre. La constitution du gouvernement n'est cependant plus militaire. Le souverain ne se dit plus Autocrate; \& l'univers, en général, est trop éclairé pour admettre cette forme odieuse*.»

* Note de Mercier : «Qui eut dit, il y a quatre-vingts ans, qu'on porteroit à Pétersbourg nos modes, nos perruques, nos brochures, nos opéra-comiques, auroit passé à coup sûr pour extra- 
un tableau de la capitale dans le futur qui nous est proposé, et non celui d'une ville ou d'un pays imaginaire (le Voyage en Ophirie de M. Ščerbatov [1784] n'a été publié qu'en 1896). Notons qu'Ulybyšev n'est pas le seul décembriste utopiste : F. Glinka, sentimentaliste, W. Küchelbecker, pessimiste ou satirique, exprimeront leurs rêves ou leurs inquiétudes sous forme de fragments ou de récits utopiques ${ }^{23}$.

Le rêve d'Ulybyšev est brutalement interrompu par l'irruption de la réalité grossière (« les hurlemens d'un paysan ivre, qu'on trainoit à la police »). Son « accomplissement est encore loin », mais cela ne veut toutefois pas dire que le récit d'Ulybyšev est « satirique » et qu'il représente la « seule utopie du XIX siècle avec un avenir peu réconfortant $»^{24}$ : celles-ci sont au contraire nombreuses, depuis les Lettres européennes de W. Küchelbecker (1819) et sa « terre des Acéphales » (1824), les utopies satiriques de F. Bulgarin (1824-1825), jusqu'aux anti-utopies de N.D. Fëdorov et de V. Brjusov en passant par les anti-utopies apocalyptiques de V. Odoevskij (également grand musicologue comme Ulybyšev, qu'il fréquentait), ou Le Songe d'un homme ridicule de Dostoevskij (1877).

Le manuscrit du «Rêve » se présente sur quatre grandes feuilles pliées en deux (35 x $22 \mathrm{~cm})$, de couleur bleue, écrites recto-verso. L'encre a pâli et le papier est taché. Nous conservons l'orthographe et la ponctuation d'Ulybyšev, et indiquons la pagination entre barres inclinées $(r=$ recto, $v=$ verso $)$.

\section{Un rêve}

De tous les genres de superstitions, celle qui prétend expliquer les rêves me paroit la plus excusable. Ils ont en effet quelque chose de mystérieux, qui nous fait reconnoitre dans leurs images fantastiques, des avertissemens du ciel, ou le symbole de notre avenir. L'ambitieux à peine livré au sommeil, qui longtemps a fui sa paupière, se voit décoré du ruban qui a causé son insomnie et se persuade à son réveil que les fêtes de Pâques ou bien le nouvel an, amèneront l'accomplissement de son rêve. L'amant malheureux jouit en songe de l'objet de sa longue convoitise et l'espérance presque éteinte se ranime dans son coeur. Bienheureuse faculté de se repaitre d'illusions, tu es le contrepoids des maux réels dont notre vie est sans cesse assiégée ; mais tes prodiges ne sont pas l'aliment l'aliment exclusif de passions

vagant. Il faut consentir paisiblement à passer pour fou, lorsqu'on a quelque idée qui surpasse l'horison des idées vulgaires. Tout en Europe tend à une révolution soudaine. »

(Édition de 1786, s.1., s. n., t. 3, p. 61-62). On verra qu'Ulybyšev rejette l'imitation exclusive de l'Occident : le costume des habitants du «Rêve » « réunissoit l'élégance Européenne à la majesté Asiatique ».

23. Heller, Niqueux, Histoire de l'utopie en Russie, p. 110-115.

24. V. Mil’don, Sanskrit vo 1'dah, ili vozvraščenie iz Ofira. Očerk russkoj literaturnoj utopii i utopičeskogo soznanija [Le sanscrit dans les glaces, ou le retour d'Ophir. Essai sur l'utopie littéraire russe et la conscience utopique], M. : ROSSPÈN, 2006, p. 29. 
égoistes. Le patriote, l'ami de la raison, le philantrope surtout ont également leurs chimères, qui se reproduisant quelquefois dans leurs songes, leur procurent quet= ques des instans d'un bonheur imaginaire, mille fois préférable à tout ce que leur offre la triste réalité. Tel est le rêve que j'ai eu la nuit dernière ; il s'accorde trop avec les désirs et les voeux unanimes de mes respectables collègues de la Lampe verte pour ne pas leur en faire part.

/1v/ Je croyois être au milieu des rues de $S^{t}$ Petersbourg, mais tout me paraissoit tellement changé que j'avois peine à m'y reconnoitre. De nouveaux édifices publics attiroient mes regards à chaque pas et les anciens sembloient consacrés à des usages qui contrastoient singulièrement avec leur destination primitive. Sur le frontispice du chateau de $\mathrm{S}^{\mathrm{t}} \mathrm{Michel}^{25}$, je lus en grosses lettres d'or : Palais de l'assemblée des $\underline{\text { états }}^{26}$. Des écoles publiques, des académies, des bibliothèques de toute espèce occupoient la place des inombrables casernes dont la ville étoit anciennement remplie. En passant devant le palais d'Аничковъ, j'apperçus à travers de grands carreaux de glace, une foule de monumens superbes en marbre et en bronze. On m'apprit que c'étoit le Panthéon Russe, out c'est-à-dire une collection de statues et bustes représentant les hommes qui se sont illustrés par leurs talens ou les services rendus à la patrie. J'y cherchais vainement l'image du propriétaire actuel de ce palais $^{27}$. Me trouvant sur la perspective de Newsky, mes yeux se portèrent en ligne droite dans un lointain immense, et au lieu du couvent qui la termine ${ }^{28}$, je vis un arc de triomphe, élevé comme sur les ruines du fanatisme.

/2r/ Tout à coup mon oreille est frappée d'une suite d'accords dont l'harmonie et la force inconnues, semblèrent être un mélange de l'orgue, de l'harmonica ${ }^{29}$ et du

25. Mihajlovskij zamok, palais de Paul $\mathrm{I}^{\text {er }}$, où il fut assassiné dans la nuit du 11 au 12 mars 1801.

26. États au sens de catégories sociales ou de nations d'un État fédéral ? Dans la traduction russe, on a gosudarstvennoe sobranie.

27. Le «propriétaire actuel » était le fils de Paul I ${ }^{\mathrm{er}}$, le grand-prince Nikolaj Pavlovič, que personne ne donnait à l'époque pour futur tsar (Nicolas ${ }^{\text {er }}$ ). Selon Modzalevskij («K istorii "Zelenoj lampy" », p. 53), Ulybyšev a sans doute plutôt en vue le successeur de Paul Ir Alexandre $\mathrm{I}^{\mathrm{er}}$.

28. La laure Saint-Alexandre-Nevski.

29. Cf. la note de George Sand sur l'harmonica dans la Comtesse de Rudolstadt (II, XLI) : « Tout le monde sait que l'harmonica fit une telle sensation en Allemagne à son apparition, que les imaginations poétiques voulurent y voir l'audition des voix surnaturelles, évoquées par les consécrateurs de certains mystères. Cet instrument, réputé magique avant de se populariser, fut élevé pendant quelque temps, par les adeptes de la théosophie allemande, aux mêmes honneurs divins que la lyre chez les Anciens, et que beaucoup d'autres instruments de musique chez les peuples primitifs de l'Himalaya. Ils en firent une des figures hiéroglyphiques de leur iconographie mystérieuse. Ils le représentaient sous la forme d'une chimère fantastique. Les néophytes des sociétés secrètes, qui l'entendaient pour la première fois, après les terreurs et les émotions de leurs rudes épreuves, en étaient si fortement impressionnés, que plusieurs tombaient en extase. Ils croyaient entendre le chant des puissances invisibles, car on leur cachait l'exécutant et l'instrument avec le plus grand soin. Il y a des détails extrêmement curieux sur le rôle extraordinaire de l'harmonica dans les cérémonies de réception de l'illuminisme. » 
serpent $^{30}$. Bientot je vis un peuple inombrable affluer vers l'endroit d'où venoient ces sons ; je me joignis à la foule et après avoir marché quelque temps, je me trouvai en face d'une rotonde dont la grandeur et la magnificence surpassoient non seulement tous nos édifices modernes, mais même les monumens gigantesques de la grandeur Romaine, dont nous ne voyons plus que les débris. Des portes de bronze, d'une élévation prodigieuse, s'ouvrirent pour recevoir la foule ; j'entrai avec les autres. La noble simplicité du dedans, répondoit à la majesté du dehors. L'intérieur de la coupole soutenue par un triple rang de colonnes représentoit la voute céleste avec ses constellations ${ }^{31}$. Au milieu de l'enceinte s'élevoit un autel de marbre blanc, sur le quel brûloit une flamme inextinguible. Le profond silence qui régnoit dans l'assemblée, le recueillement empreint sur toutes les figures, me firent juger que j'étois dans un temple, mais de quelle religion, voilà ce que je ne pouvois deviner. Point de statue ou d'image, point de prêtre dont le costume et les gestes puissent fixer mes doutes ou diriger mes conjectures. Après quelques moments d'un silence préparatoire, plusieurs voix d'une justesse et d'un timbre admirables $/ 2 \mathrm{v} /$ entonnèrent un chœur de la création. Il me parut que l'exécution répondoit pour la première fois à la sublimité du génie de Haydn et je crus assister effectivement au concert des Anges $^{32}$. Il devoit y avoir, par conséquent, des voix de femmes ? sans doute, et cette nouveauté aussi conforme au bon gout qu'à la raison, me causa un plaisir inexprimable : car me disois-je puisque l'insecte par son bourdonnement et l'oiseau par son ramage célèbrent la gloire du Très haut, par quelle injustice ridicule et barbare a-t-on défendu à la moitié la plus intéressante du genre humain, de chanter ses louanges ${ }^{33}$. Les sons ravissans de cette musique, se mêlant aux vapeurs des parfums

30. Serpent : sorte d'instrument à vent, en forme de serpent, en bois et couvert de cuir, qui était employé autrefois pour accompagner les chantres à l'église.

31. Les étoiles sont aussi représentées sur l'intérieur du grand dôme du temple circulaire de La ville du soleil de Campanella. Mais il est peu probable qu'Ulybyšev ait pu lire ce texte : les éditions étaient rares, et uniquement en latin et en italien. Par contre, il connaissait certainement $L$ 'an 2440, rêve s'il en fut jamais (1771) de Louis-Sébastien Mercier, dont voici la description du temple (chap. XIX) : «J'apperçus au milieu d'une belle place un temple en forme de rotonde, couronné d'un dôme magnifique. Cet édifice soutenu sur un seul rang de colonnes avoit quatre grands portails. [...] Point de statues, point de figures allégoriques, point de tableaux. [...] Si on levoit les yeux vers le sommet du temple, on voyoit le ciel à découvert ; car le dôme n'étoit pas fermé par une voûte de pierre, mais par des vitraux transparens. »

Modzalevskij («K istorii "Zelenoj lampy” », p. 57) indique que la description du temple d'Ulybyšev rappelle celle du temple franc-maçon dans La comtesse de Rudolstadt de George Sand (livre II, chap. XLI), mais ce roman date de $1843 \ldots$ : « En ce moment les portes du temple s'ouvrirent en rendant un son métallique, et les Invisibles entrèrent deux à deux. La voix magique de l'harmonica, cet instrument récemment inventé, dont la vibration pénétrante était une merveille inconnue aux organes de Consuelo, se fit entendre dans les airs, et sembla descendre de la coupole entrouverte aux rayons de la lune et aux brises vivifiantes de la nuit. » Ulybyšev n'a certainement pas été franc-maçon. J.-V. Hocquard regrette que dans son ouvrage sur Mozart, Ulybyšev ne mentionne qu' « à la sauvette » l'appartenance de Mozart à la Maçonnerie (A. Oulibicheff, Mozart. Préface de Jean-Victor Hocquard. P. : Librairie Séguier, 1991, p. 10).

32. Il s'agit de l'oratorio de Haydn « La création » (1796-1798).

33. «L'usage de chanter les psaumes par les deux sexes fut aboli en 379 par le synode d'Antioche » (F.-J. Fétis, Histoire générale de la musique depuis les temps les plus anciens jusqu'à nos jours, t. IV, P. : Didot, 1874, p. 7). L'interdiction faite aux femmes de chanter dans 
qui brulaient sur l'autel, montoient vers les hauteurs immenses de la voute et sembloient être le dépot des pensées pieuses, des élans de reconnoissance et d'amour, qui s'échappoient de tous les coeurs vers la divinité. Enfin les chants cessèrent ; un vieillard revetu de plusieurs marques de distinction qui m'étoient inconnues, monta vers les dégrés de l'autel et prononça ces paroles : «Citoyens, en portant l'hommage de notre reconnoissance au dispensateur de tous les biens, nous avons rempli un devoir sacré ; mais ce devoir ne seroit qu'une vaine formule, si n'honorions également la divinité par nos oeuvres. Ce n'est qu'en nous conformant aux loix de l'humanité, $/ 3 \mathrm{r} /$ aux sentimens de commisération envers nos frères malheureux, que Dieu lui-même a gravés dans nos âmes, que nous pouvons espérer une éternité de bonheur, pour prix de quelques années de vertu. » Après avoir ainsi parlé, le vieillard recommanda à la charité des assistans plusieurs pauvres, dont les circonstances seules avoient occasionné la détresse et qui n'avoient rien fait pour la mériter. Chacun s'employa de contribuer d'après ses moyens, et dans l'espace de quelques minutes, je vis une somme suffisante pour tirer dix familles de l'indigeance $^{34}$. J'étois confondu de tout ce que je voyois et par une de ces inconséquences inexplicables, mais très fréquentes en songe, j'oubliai tout à coup mon nom, mon pays et me crus un étranger arrivé pour la première fois à $S^{t}$ Petersbourg. M'étant approché du vieillard, que malgré sa haute dignité,j'abordai sans obstacle : seigneur lui dis-je pardonnez à la curiosité d'un étranger qui ne sachant s'il doit croire à ses yeux, ose vous demander l'explication de tant de merveilles. Vos concitoyens ne sont-ils pas de la confession Greco Catholique, mais l'auguste réunion dont je viens d'être le témoin, ne ressemble pas plus à une messe Grecque que Latine, et n'offre même aucune trace de chrétienté. Et d'où venez vous donc me répondit le $/ 3 \mathrm{v} /$ vieillard. L'etude de l'histoire vous a-t-elle absorbé au point de ressusciter le passé et de faire disparaitre l'avenir le présent devant vos yeux? Il y a près de trois siècles que la vraie religion, c'est-à-dire que le culte d'un dieu unique et tout puissant, fondés sur le dogme de l'immortalité de l'âme, des peines et des récompenses après la mort, mais dégagé de tout alliage humain ou de toute croyance superstitieuse, a été établi parmi nous ${ }^{35}$. Ce n'est point à un gateau de froment, ni au gui du chêne, ni

les églises (sauf dans les monastères de femmes) se fonde sur saint Paul (1 Co 14,34) interprété étroitement. Au XIX ${ }^{\mathrm{e}}$ siècle, tous les chœurs professionnels étaient masculins, bien que quelques compositeurs (A.F. L'vov, D.S. Bortnjanskij) aient écrit des partitions de prières pour chœurs mixtes. En 1873, M.N. Katkov souhaitait que dans les églises orthodoxes, toute l'assemblée soit autorisée à chanter ( « Ogrubenie našego cerkovnogo byta. Pol’za dopuščenija vsenarodnogo penija » [« La dégradation de la vie de notre Église. De l'utilité d'admettre le chant public »], Moskovskie vedomosti, $\mathrm{n}^{\circ}$ 87, 1873, repris in Id., Imperija i kramola [L'Empire et la sédition], M. : Fond IV, 2007, p. 196-198). C'est seulement à partir du début du XXe siècle qu'apparurent des chœurs mixtes. On notera le féminisme d'Ulybyšev.

34. «Quelle que soit sa condition, en tout lieu, le membre de l'Union doit aider son prochain » (Règlement [Zakonopoloženie] de l'Union du bien public, livre 2, chap. I, § 8.

[http://decemb.hobby.ru/index.shtml?archive/blago])

35. Cf. Rousseau, Du contrat social (livre IV, chap. VIII, « De la religion civile ») : « L'existence de la Divinité puissante, intelligente, bienfaisante, prévoyante et pourvoyante, la vie à venir, le bonheur des justes, le châtiment des méchants, la sainteté du contrat social et des lois : voilà les dogmes positifs. » 
à un oignon ${ }^{36}$ etc., que nous adressons nos prières, mais à celui que le plus grand poëte d'une nation longtemps notre institutrice, a défini par un seul vers : L'éternel est son nom, le monde est son ouvrage ${ }^{37}$. Il existe encore parmi le bas peuple des vieilles et des cagots qui regrettent les anciennes cérémonies; rien ne devoit être plus beau disent-ils que de voir officier l'archevêque, de voir une douzaine de prêtres et de diacres transformés en laquais, s'occuper à sa toilette, faire des génuflexions et lui baiser la main à chaque instant pendant que lui seul est étoit assis et que les fidèles se tenoient debout. N'étoit-ce pas là je vous prie, une véritable idolatrie moins élégante que celle des anciens Grecs, mais plus ridicule, puisqu'elle identifioit l'idole le prêtre avec l'idole. A présent nous n'avons plus de prêtres et de moines encore moins. Chaque magistrat supérieur/4r/ remplit alternativement les fonctions dont je me suis acquitté aujourd'hui. En sortant du temple, je vais rendre la justice. Celui qui veille au maintien de l'ordre sur la terre n'est-il pas le plus digne représentant de dieu, qui est la source de l'ordre universel ? Rien de plus simple que notre culte. Vous ne voyez dans notre temple ni image ni statue. Nous pensons qu'une représentation matérielle de la Divinité est non pas injurieuse, mais simplement ridicule. La musique est le seul art qui puisse être admis de droit dans nos temples. Elle est le language naturel entre l'homme et la divinité, puisqu'elle fait pressentir ce qu'aucune langue ne peut exprimer, ce que l'imagination elle même ne sauroit concevoir. Mon devoir m'apelle ailleurs, observa le vieillard ; si vous voulez m'accompagner, je me ferai un plaisir de vous entretenir des changemens et des réformes qui ont eu lieu en Russie depuis $300^{38}$ ans et dont vous paroissez si peu instruit. J'acceptai son offre avec reconnoissance et nous sortimes du temple. J'avois été frappé en parcourant la ville du costume de ses habitans. Il réunissoit l'élégance Européenne à la majesté Asiatique et en l'examinant avec plus d'attention je reconnus le Kafftan Russe, à quelques modifications près. Il me semble, dis-je à mon guide, que Pierre le Grand avoit fait prendre le costume Allemand aux hautes classes de la société en Russie ; depuis quand l'avez-vous quitté ? Depuis que nous $/ 4 \mathrm{v} /$ sommes devenus une nation, répondit-il, depuis qu'ayant

36. Traduit par miro (saint-chrême) dans la version russe. Mais c'est bien oignon, et non onguent qu'il y a dans le manuscrit. Y aurait-il un jeu de mots : luk(ovica) (oignon) // lukovka (bulbe d'église)? Dans l'Antiquité, l'oignon était vénéré : «D'après Athénée, ce légume était consacré à la déesse Latone, depuis qu'étant enceinte et ayant perdu l'appétit, un oignon le lui rendit [...] D'après Pline (XIX, 101), les anciens Égyptiens prêtaient serment par l'ail et par l'oignon » (Angelo de Gubernatis, Mythologie des plantes ou les légendes du règne végétal [1882], Milan : Archè, t. 2, 1976, p. 256).

\section{Racine, Esther III, 4 :}

L'Éternel est son nom, le monde est son ouvrage.

Il entend les soupirs de l'humble qu'on outrage,

Juge tous les humains avec d'égales lois

Et, du haut de son trône, interroge les rois.

Des plus fermes États la chute épouvantable,

Quand il veut, n'est qu'un jeu de sa main redoutable

Et les faibles humains, vains jouets du trépas,

Sont tous devant ses yeux comme s'ils n'étaient pas.

38.200 corrigé en 300 , semble-t-il. 
cessé d'être esclaves, nous ne portons plus la livrée du maître. Pierre le Grand, malgré des talens extraordinaires, avoit plutôt le génie imitateur que créateur ${ }^{39}$. En faisant prendre à un peuple barbare le costume et les moeurs des étrangers, il lui a donné en peu de temps une apparence de civilisation, mais cette civilisation précoce étoit à la véritable ce que la plante éphémère d'une serre chaude est au chêne antique que l'air, le soleil et la succession des années ont élevé comme un rempart contre la foudre, ou un monument à l'éternité. Pierrre étoit trop amoureux de sa gloire pour être entièrement patriote. Il vouloit jouir pendant sa vie d'un développement qui ne pouvoit être que le résultat des siècles ${ }^{40}$. Le temps seul produit les grands hommes dans tous les genres, qui déterminent le génie d'une nation, et lui tracent la route qu'elle doit suivre. L'impulsion donnée par ce souverain retarda longtemps parmi nous les véritables progrès de la civilisation. Nos essais dans les beaux arts, calqués sur les productions des étrangers, laissèrent pendant près de deux siècles entre eux et nous la distance qui sépare l'homme et le singe. Nos ouvrages littéraires, surtout, portoient l'empreinte de la décadence, avant d'avoir atteint la maturité, et $/ 5 \mathrm{r} / \mathrm{l}$ 'on pouvoit assimiler notre littérature ainsi que nos institutions ${ }^{41}$ à un fruit verd d'un côté et pourri de l'autre ${ }^{42}$. Heureusement nous nous apperçumes de notre égarement. Les grands evenemens qui en brisant nos fers, nous ont élevé au premier rang des peuples de l'Europe, ont également ranimé l'étincelle presqu'éteinte de notre génie national. On a fouillé la veine féconde et presqu'intacte de nos antiquités et de nos traditions populaires et bientot cette veine a fait jaillir le feu poétique, qui brille aujourd'hui avec tant d'éclat, dans nos épopées et nos tragédies. Les mœurs prennent de plus en plus ces traits caractéristiques qui distinguent toujours les nations libres, ont donné naissance à la bonne comédie, à la comédie originale, parmi nous $^{43}$. Nos presses ne sont plus occupées à reproduire et à augmenter le tas inutile

39. Cf. Rousseau, Du contrat social (livre II, chap. 8, « Du peuple ») : « Pierre avait le génie imitatif ; il n'avait pas le vrai génie, celui qui crée et fait tout de rien. [...] Il a d'abord voulu faire des Allemands, des Anglais, quand il fallait commencer par faire des Russes. » Le point de vue d'Ulybyšev sur Pierre le Grand est très proche de celui exposé par Karamzin dans son mémoire de 1811 sur « La Russie ancienne et moderne » («O drevnej i novoj Rossii »), qui n'était pas encore connu du public : «Nous sommes devenus des citoyens du monde, mais, dans certains cas, nous avons cessé d'être des citoyens de la Russie. La faute en incombe à Pierre. »

40. Cf. Montesquieu, L'esprit des lois (livre XIX, 14 [chapitre sur la « Moscovie »]) : « En général, les peuples sont très attachés à leurs coutumes; les leur ôter violemment, c'est les rendre malheureux : il ne faut donc pas les changer, mais les engager à les changer eux-mêmes. »

41. ainsi que nos institutions : suscrit.

42. Cf. Diderot, «Sur la civilisation de la Russie », in Contributions à l'Histoire des deux Indes, chap. III : «Un observateur étranger qui avait parcouru la plus grande partie de l’empire, disait-il que le Russe était pourri avant d'avoir été mûr » (Diderot, Euvres (éd. L. Versini), Robert Laffont («Bouquins »), t. 3, 1995, p. 663).

43. Allusion possible aux comédies de Krylov, de Šahovskoj. Les comédies de A. Griboedov (collègue en 1817-1818 d'Ulybyšev au Collège des Affaires étrangères) d'avant Le malheur d'avoir de l'esprit (1824), Molodye suprugi [Les jeunes époux, 1814], Pritvornaja nevernost' [L'indidélité feinte, 1818], furent critiquées par les « lampistes » pour être des imitations de pièces françaises (M.V. Nečkina, A.S. Griboedov i dekabristy [A.S. Griboedov et les décembristes], M. : Izd-vo AN SSSR, 1951, p. 185). Les « lampistes » étaient de grands amateurs de théâtre, et écrivaient eux-mêmes des pièces qu'ils mettaient en scène chez N. Vsevoložskij. 
de nos traductions de pièces françaises, vieillies chez le peuple même pour le quel elles furent composées. Ainsi ce n'est qu'en nous éloignant des étrangers à l'exemple des toutes les nations écrivains de tout pays, qui ont créé chez eux une littérature nationale, que nous sommes parvenus à les égaler, et qu'après avoir été leurs vainqueurs par les armes, nous sommes devenus leurs alliés par le génie ${ }^{44}$ !!!

Pardon si je vous interromps, Seigneur, mais je ne vois pas cette foule de militaires, dont votre ville m'avoit-on dit, étoit le principal dépot. Nous avons pourtant plus de soldats répondit-il, qu'il n'y en a jamais eu en Russie /5v/ car leur nombre s'élève à 50 millions d'hommes. Comment une armée de 50 millions d'hommes, vous plaisantez, Seigneur! Rien n'est plus vrai, car la nation et l'armée ne sont qu'une seule et même chose. Chaque citoyen devient un héros devient un héros, lorsqu'il s'agit de défendre le terrain qui le nourrit, les loix qui le protègent, les enfans qu'il élève dans les principes de la liberté et de l'honneur, et la patrie dont il se fait gloire d'être fils. Nous n'entretenons plus il est vrai ces inombrables essaims de fainéans et de voleurs enrégimentés, fléau du peuple qui les nourrit, autant que de ceux contre lesquels on les envoie et qui s'ils ne détruisent les générations par le feu, en infectent la source par les maladies contagieuses ${ }^{45}$. Ils ne nous sont plus nécessaires. L'échaffaudage qui soutenoit le despotisme, a croulé avec lui. L'amour et la confiance du peuple, et surtout les loix qui otent au souverain la possibilité d'abuser de son pouvoir, forment autour de sa personne une garde plus sure que 60 mille bayonnettes. Je vous demande d'ailleurs si les républiques anciennes les plus célèbres par leurs exploits militaires, comme Sparte, Athènes et Rome, avoient des troupes réglées. Le service intérieur-nécessaire à la sureté intérieure de l'état du pays est successiveemnt rempli par tous les citoyens en état de porter les armes, sur toute l'étendue de l'empire. Vous concevez que ce changement dans le système militaire en a produit aussi un très grand dans celui des finances. Les trois quarts /6r/ de nos revenus, anciennement absorbés par l'entretien de l'armée, qui n'en mouroit pas moins de faim, sont employés à présent à augmenter la prospérité publique, en encourageant l'agriculture, le commerce et l'industrie, et en soulageant les pauvres dont le nombre grâce au ciel diminue tous les jours sous le gouvernement paternel de la Russie. Dans ce moment nous nous trouvames au milieu de la place du palais. L'ancien drapeau flottoit encore sur ses murs noirs de vétusté, mais au lieu de

44. Cf. la fin de la «Lettre à un ami d'Allemagne sur les sociétés de Pétersbourg » (f. 244, op. $\left.36, \mathrm{n}^{\circ} 41,1.5\right)$ : «C'est donc en exploitant nos propres richesses dont les étrangers ont su profiter plutôt que nous-mêmes, et non point en glanant pitoyablement sur les champs d'autrui, que nous pourrions un jour rivaliser avec les Français et qu'après leur avoir arraché les lauriers de Mars, nous pourrons leur disputer encore ceux d'Apollon. »

45. Cette phrase est coupée (à partir de « autant ») et remplacée par des points de suspension dans l'édition de Russkaja literaturnaja utopija, p. 91. Dans Izbrannye social'no-političeskie $i$ filosofskie proizvedenija dekabristov, p. 291, une note indique que Ulybyšev fait allusion aux colonies militaires d'Arakčeev. Selon Gaciskij (« K istorii “Zelenoj lampy” », p. 58), Ulybyšev traduisit en français pour le gouvernement une brochure sur les colonies militaires. Dans ses Considérations sur le gouvernement de Pologne et sur sa réformation projetée en avril 1772, Rousseau rejetait les «troupes réglées, peste et dépopulation de l'Europe », et donnait en exemple la Suisse, où « tout habitant est soldat, mais seulement quand il faut l'être » (chap. XII, « Système militaire »). 
l'aigle à deux têtes tenant la glaive foudre dans ses griffes, je vis un phoenix planant dans les nues et tenant dans son bec une couronne d'oliviers et d'immortelles. On a changé les armes de l'empire comme vous le voyez me dit mon conducteur. Les deux têtes de l'aigle qui signifiaient le despotisme et la superstition ont été abbatues et du sang qui en a jailli est sorti le phoenix de la liberté et de la vraie croyance ${ }^{46}$.

Arrivés sur le grand quai de la Néwa, je vis en face du palais un pont magnifique, moitié de granit et moitié de marbre ${ }^{47}$, qui conduisoit à un édifice superbe ${ }^{48}$ situé de l'autre coté de la rivière et sur le fronton du quel je lus : Le sanctuaire de la justice est ouvert à chaque citoyen et il peut réclamer à toute heure la protection des loix. C'est là me dit le vieillard que s'assemble le tribunal suprême, composé des anciens de la nation, dont j'ai l'honneur d'être membre. Je me préparois à passer le pont lorsque $/ 6 \mathrm{v} /$ le bruit du fiffre et du tambour, et les hurlemens d'un paysan ivre, qu'on trainoit à la police, me réveillèrent en sursaut. Je pensai que l'accomplissement de mon rêve étoit encore loin.

\section{$* * *$}

\section{Après l'utopie : le réformisme}

«Un rêve » est une œuvre de jeunesse. Ulybyšev mène ensuite, on l'a vu, une belle carrière sous Nicolas $\mathrm{I}^{\text {er }}$ : il fut même chargé de rédiger la relation du couronnement de Nicolas I ${ }^{\mathrm{er}}$, pour laquelle il reçut les diamants de l'ordre de Sainte-Anne $\left(2^{\mathrm{e}} \text { classe }\right)^{49}$. Il a renoncé à tout changement révolutionnaire, si tant est qu'il l'ait jamais souhaité, comme il semble découler de sa critique de Pierre le Grand. À la fin de son livre sur Beethoven (1857) dont il va être question, Ulybyšev déplore la « décomposition des idées religieuses, philosophiques et sociales, à laquelle l'Allemagne a été en proie, pendant le deuxième quart de notre siècle et dont l'année 1848 est venue mûrir et répandre les détestables fruits $\gg^{50}$. Il n'est plus attiré par l'utopie de Platon, à supposer qu'il le fût jamais : toujours à propos de Beethoven, il écrit : «Pour ce qui est des opinions politiques du grand artiste, elles étaient plus que singulières. Il considérait la république de Platon comme le

46. Nečkina estime que cela « témoigne d'une révolution qui fut loin d'être paisible » (« Dekabristskaja "Utopija”... », p. 381). Ulybyšev dit pourtant, en critiquant Pierre le Grand : «Il voulait jouir pendant sa vie d'un développement qui ne pouvoit être que le résultat des siècles. »

47. Le Pont du palais (pont ouvrant) sera construit en 1912-1916. Jusqu'en 1851, date de la construction du pont de l'Annonciation (plus tard Nikolaevskij, plus tard Lieutenant Šmidt), le Dvorcovyj most était un pont en bois démontable.

48. superbe : suscrit.

49. Gaciskij, « K istorii "Zelenoj lampy” », p. 58.

50. Alexandre Oulibicheff, Beethoven ses critiques et ses glossateurs, Leipzig : Brockhaus, 1857, p. 332. 
modèle de tous les gouvernements à établir dans les cinq parties du monde ; sorte d'idée fixe qui le domina toute sa vie et sur laquelle il ne souffrit jamais la moindre contradiction. ${ }^{51} \mathrm{Il}$ ironise sur l'utopie wagnérienne exprimée dans das Kunstwerk der Zukunft (L'œuvre d'art de l'avenir, 1850), ouvrage qui est dédié à Feuerbarch : « Il est évident que l'homme du docteur Feuerbach vaut un million de fois plus que l'homme du bon Dieu. Or, l'homme atteindra infailliblement aux susdites perfections et à bien d'autres encore, du moment où il n'y aura plus de religion, de gouvernement, de propriété, d'état, de nationalité, ni de famille ${ }^{52}$. Il critique (toujours dans son ouvrage sur Beethoven) « les philosophes qui rejettent l'idée de Dieu, cette base éternelle de la raison humaine », « les révolutionnaires saisis du vertige de l'athéisme et du communisme, les zélateurs de l'impossible, [qui] ont sympathisé avec une musique impossible, et se sont reconnus dans le désordre d'une inspiration que l'âge et les souffrances avaient affaiblie, que l'oreille ne guidait plus depuis dix ans, et que d'étranges hallucinations achevèrent de vicier dans sa source $»^{53}$. «Il est évident que ce sont les infirmités morales et physiques du grand artiste qui l'ont transformé en précurseur d'une nouvelle ère de l'humanité, en apôtre du socialisme démocratique et de l'athéisme, en une idole symbolique, figurant les rêves les plus insensés et les plus criminelles tentatives des révolutionnaires et émeutiers allemands de 1848. ${ }^{54}$ La musique, pour Ulybyšev, ne saurait prendre aucune couleur politique ou philosophique.

Est-ce à dire que Ulybyšev est devenu un réactionnaire? Nullement. Le témoignage de son biographe de Nižnij Novgorod, A. Gaciskij, les quelques pages retrouvées de son Journal (dont nous donnons de larges extraits dans la publication de sa lettre sur les sociétés de Saint-Pétersbourg) ${ }^{55}$, nous montrent un homme à l'esprit critique qui une fois libre de toute tutelle administrative ne craint pas d'être dans l'opposition au gouverneur militaire de Nižnij Novgorod (M.A. Urusov), fustige dans des pièces satiriques (et dans son Journal) l'arrivisme et la concussion des « sommités » de sa ville, participe à la fin des années 1850 au comité de la province « pour l'amélioration de la vie des paysans » et se prononce pour l'abolition du servage $^{56}$. En somme, il observe le point 11 (livre 2) du règlement de l'Union du bien public, qui stipulait que chacun de ses membres « doit par son exemple personnel et sa parole encourager tout un chacun à la vertu, répandre les idées conformes aux buts de l'Union, dire la vérité et lutter sans crainte pour celle-ci ». Le but de l'Union était la prospérité de la nation, par la pratique de la philanthropie, le développement de la moralité et de l'éducation, l'amélioration de la justice et

51. Id., p. 68.

52. Id., p. 323.

53. Id., p. 347-348.

54. Id., p. 316.

55. M. Aronson, « Zapiski A.D. Ulybyševa » [Journal de A. D. Ulybyšev], Zvezda, 3, 1935, p. 174-197.

56. Cf. Gaciskij, « A.D. Ulybyšev », p. 68 ; Aronson, «Zapiski A.D. Ulybyševa », p. 176. 
de l'économie. Devenu un libéral éclairé, Ulybyšev reste « le patriote, l'ami de la raison, le philanthrope » qu'était le narrateur du « Rêve ».

Ulybyšev fréquente aussi assidûment le théâtre local (quatre fois par semaine) et ses jugements, lancés au cours du spectacle, ou ses bons mots, font le tour de la ville. Il écrit lui-même des pièces (drames, comédies, satires des mœurs provinciales), sans intention de les publier, mais qu'il fait jouer chez lui. Une seule pièce a été publiée après sa mort (en 1886, à la suite de la biographie de Gaciskij) : Raskol'niki : Istoriko-bytovaja drama v 5 dejstvijah [Les schismatiques : Drame de mœurs historique en cinq actes]. Le principal intérêt de ce texte didactique sans grand ressort dramatique (écrit en 1850) est la dénonciation de la persécution des vieux-croyants, à travers l'exposé d'un paysan sur les causes et de la nature des différentes sectes. Gérant pendant vingt ans d'un domaine de 18000 âmes, il a luimême été doukhobor avant de professer un christianisme « selon le Christ » (et non selon l'Église), en rejetant dogmes et sacrements (Trinité, eucharistie, mariage). Il expose ses idées sur la religion et l'État (derrière lesquelles on perçoit celles d'Ulybyšev utopiste) à un « Inconnu » qui s'avère être un membre du Conseil d'État venu se renseigner sur les sectes (Ulybyšev tenait sa documentation des enquêtes de N.I. Nadeždin sur le raskol ${ }^{57}$, que lui avait communiquées V. Dal', lui aussi habitant de Nižnij Novgorod). Ce texte témoigne de l'intérêt qu'Ulybyšev continue de porter à la religion (il avait des discussions religieuses avec son économe aux vues conservatrices $)^{58}$.

A. Gaciskij ne dit mot de l'appartenance d'Ulybyšev à La Lampe verte. Sans doute l'ignorait-il, et Ulybyšev ne devait-il pas s'en targuer. Mais la critique, dans Raskol'niki, de l'Église soumise au pouvoir temporel et des dogmes, la dénonciation du servage, des abus des fonctionnaires, de la censure constituent un réquisitoire qui montre la permanence de ses idéaux décembristes, à l'époque de Nicolas ${ }^{\text {er }}$.

Ulybyšev était un homme rempli des aspirations les plus élevées et les plus humanistes, qui devinait intuitivement et aimait ardemment le beau dans l'art, qui haïssait profondément le mensonge dans les relations humaines et dans l'ordre social [...] Il dénonçait le servage, l'arbitraire administratif, l'exploitation des schismatiques par la police, etc. ${ }^{59}$

57. Deux des rapports de Nadeždin ont été publiés : Issledovanie o skopčeskoj eresi [Enquête sur l'hérésie des castrats], SPb. 1845, O zagraničnyx raskol'nikax [Sur les schismatiques établis à l'étranger], 1846. Réédités dans Sbornik pravitel'stvennyx svedenij o raskol'nikax [Recueil des documents gouvernementaux sur les schismatiques] de L. Kel'siev (vyp. 1-4, Londres, 1860-1862).

58. Gaciskij, «A.D. Ulybyšev », p. 65

59. «O žizni i trudah Ulybyševa » [« De la vie et des travaux d'Ulybyšev »], in G. Laroš, Novaja biografija Mocarta A.D. Ulybyševa. Perevod M. Čajkovskogo s primečanijami G. Laroša [Nouvelle biographie de Mozart par A.D. Ulybyšev. Traduction de M. Čajkovskij, notes de G. Laroš [Laroche]], M. : L. Jurgenson, 1890, t. 1, p. 19. 


\section{La musique comme utopie}

On a vu l'importance de la musique dans la cérémonie religieuse d' « Un rêve ». Dans l'ouvrage qu'il consacrera à Mozart, Ulybyšev se dit «musicien dès l'âge de sept ans, jouant passablement du violon, chanteur au besoin, initié aux principes de la composition, enfin membre honoraire de la société philharmonique de Pétersbourg [depuis 1827] en récompense de quelques articles d'amateur publiés dans les journaux ${ }^{60}$. Selon Gaciskij, Ulybyšev publia dans le Journal de St.-Pétersbourg nombre d'entrefilets sur la vie musicale de Saint-Pétersbourg. Dans la mesure où les articles du Journal de St.-Pétersbourg ne sont pas signés, il est difficile d'attribuer à Ulybyšev toutes les annonces de concerts ou les comptes rendus que l'on y trouve ${ }^{61}$. Mais une "Revue musicale ", présentée comme un «article communiqué », dans le ${ }^{\circ} 36$ de 1839 , et, dans le n ${ }^{\circ} 48$, la «Biographie d'un jeune artiste » (le violoniste Henri Vieux-Temps, né en 1820 en Belgique, qui deviendra l'un des virtuoses et des compositeurs les plus célèbres du XIX ${ }^{e}$ siècle $^{62}$ ), portent bien la marque d'Ulybyšev, lui-même violoniste et découvreur de talents. Plus tard (1850-1854), il enverra des articles de critique musicale à Severnaja pčela.

À Nižnij Novgorod, Ulybyšev lit beaucoup, et en particulier la biographie de Mozart par Nissen (parue en allemand en 1828), qui lui donne l'idée de trier et de mettre en ordre ces matériaux bruts et de les enrichir d'une analyse des œuvres de Mozart. Il pensait que ce travail lui prendrait trois ou quatre mois, mais de nombreuses études et lectures se révélèrent nécessaires, et c'est le fruit d'un labeur de dix ans qui vit le jour à Moscou en $1843^{63}$ :

Des voyages, des affaires de toute espèce, les soins d'une exploitation agricole assez considérable m'ont souvent fait déposer la plume et souvent pour plusieurs mois ; mais l'idée de Mozart ne cessa pas un instant de me poursuivre à travers les récoltes de seigle et d'avoine; elle courait les grands chemins avec moi et, au retour, elle était la première à m' accueillir sur le seuil de mes pénates ${ }^{64}$

60. Oulibicheff, Mozart, p. 20.

61. Voir une petite bibliographie des articles de critique musicale d'Ulybyšev dans l'Encyclopédie musicale (http://enc-dic.com/enc_music/Ulybyshev-A-D-7336.html). Dans une lettre à P.A. Vjazemskij du 22 mai 1825, A.I. Turgenev indique que l'article en français sur « Černec » (« Le moine », 1824, poème de I.I. Kozlov) paru dans la Gazette de Pétersbourg (Sanktpeterburgskaja gazeta, ${ }^{\circ}$ 61, 66, 1825) est dû à Ulybyšev (Ostaf'evskij arxiv, t. III, SPb., 1899, p. 126).

62. De 1846 à 1853, Vieuxtemps (V'ëtan) sera soliste au Théâtre impérial de Saint-Pétersbourg. Il mourut en 1881 .

63. Nouvelle biographie de Mozart, suivie d'un aperçu sur l'histoire générale de la musique et de l'analyse des principales æuvres de Mozart, par Alexandre Oulibicheff, membre honoraire de la société philharmonique de Saint-Pétersbourg, M.: imprimerie d'Auguste Semen, 1843, 3 vol. (édition à compte d'auteur). La réédition citée plus haut (note 61) comporte un seul volume de 750 pages.

64. Oulibicheff, Mozart, p. 20. 
En 1841, Ulybyšev put former chez lui, à Nižnij Novgorod, un quintette à cordes, élargi à d'autres musiciens (militaires) et à des chantres mis à sa disposition par l'évêque de Nižnij Novgorod. Il y tient le premier violon, avec de bons amateurs, dont le « colonel Vertowsky ${ }^{65}$. Cela permit à Ulybyšev de jouer nombre de partitions qu'il ne pouvait auparavant que lire et entendre de tête. Il remarque et soutient le talent du jeune Balakirev, né à Nižnij Novgorod en 1836, à qui il ouvre (et lèguera) sa riche bibliothèque musicale et qu'il présentera à $\mathrm{M}$. Glinka à SaintPétersbourg en $1855^{66}$.

Il écrivit son Mozart en français, d'une part parce que «notre langue russe, si belle et si riche, mais non encore achevée, présente de nombreuses lacunes dans presque toutes les terminologies scientifiques », et d'autre part parce que pour un ouvrage d'histoire et d'analyse musicale en russe, le cercle des lecteurs n'aurait guère dépassé celui des amis et connaissances, « une cinquantaine de personnes au plus ${ }^{67}$. Il sera traduit en allemand, en suédois, et en russe par Modest Čajkovskij ${ }^{68}$. La préface de cette traduction, écrite par German Laroš (1845-1904), fils d'un enseignant de français (Laroche), professeur au Conservatoire de Moscou (18671870) puis à celui de Saint-Pétersbourg (1872-1879) et critique musical conservateur, manifeste un parti pris hostile dû peut-être au sentiment de supériorité du musicologue professionnel sur le « dilettante » qu'était Ulybyšev. Laroche trouve que le livre est écrit « dans un mélange de nijni-novgorodien et de français ». Cette critique est dénuée de tout fondement : l'ouvrage a été réédité en France en 1991 sans avoir été ni corrigé ni élagué, et son préfacier (Jean-Victor Hocquard ${ }^{69}$ ), qui d'emblée le désigne comme « l'ouvrage le plus important qui ait été écrit en français au XIX ${ }^{\mathrm{e}}$ siècle sur Mozart », ne tarit pas d'éloges sur la langue d'Ulybyšev : « Le français dans lequel il écrit est non seulement correct, mais d'une distinction littéraire si haute, que c'est un vrai régal de le lire $»^{70}$. Laroche poursuit ainsi l'éreintement d'Ulybyšev : «Il raconte d'une manière molle et verbeuse [...] on ne voit nulle part de marques d'un esprit profond et souple ». À quoi semble répondre J.-V. Hocquard, qui tout en reconnaissant que le livre « comporte des passages caducs, où la pensée est très diluée », affirme : « Cet ouvrage, à côté de ces passages

65. Vasilij Nikolaevič Verstovskij, frère du compositeur A.N. Verstovskij (1799-1862).

66. Cf. P.D. Boborykin, Za polveka : Vospominanija [En un demi-siècle : Souvenirs], M. : Zaharov, 2003, p. 25,67, 268. Boborykin et Balakirev furent camarades de classe; V.V. Stasov, Izbrannye sočinenija [EEuvres choisies], M. : Iskusstvo, t. 2, 1952, p. 544 ; N. Kaškin, Očerk istorii russkoj muzyki [Précis d'histoire de la musique russe], M. : L. Jurgenson, 1908, p. 127 ; André Lischke, Histoire de la musique russe : Des origines à la Révolution, P. : Fayard, 2006, p. 239.

67. Oulibicheff, Mozart, p. 23-24.

68. Novaja biografija Mocarta A.D. Ulybyševa. La traduction allemande ( $2^{\mathrm{e}}$ édition, Stuttgart, 1859) est disponible sur le site http://www.zeno.org/Musik/M/Ulibischeff,+Alexander/ Mozart\%27s+Leben+und+Werke.

69. Musicologue, spécialiste de Mozart (1910-1995).

70. Oulibicheff, Mozart, p. 9 ; p. 10 : «Cet ouvrage est si bien écrit qu'aujourd'hui encore, il est non seulement lisible, mais plein de charme.»; p. 14 : «J'ai abondamment louangé la pureté de style de l'écrivain ». 
qui datent, présente, non moins nombreuses, des pages très fortes, d'une grande concentration de réflexion, d'une grande densité de pensée, qui le rendent encore pertinent et enrichissant de nos jours. ${ }^{71}$ Enfin, selon Laroche, ce n'est pas seulement la forme qui pèche, mais aussi la philosophie de l'auteur : pour Ulybyšev, « tous les phénomènes du monde musical [...] ne sont pas autre chose que des degrés préparatoires, destinés par le Créateur à rendre possible l'apparition de Mozart. C'est une sorte de caricature de Hegel $»^{72}$. Il est vrai qu'Ulybyšev découvre, dans la « carrière d'homme et d'artiste » de Mozart, « la direction d'une volonté supérieure $»^{73}$. Mais en fait il rejoint, sans que cela fût son point de départ, l'idéalisme esthétique de Schelling et des romantiques. Mozart est « l'idéal absolu », il fait la synthèse de tout ce qui a précédé, sa musique est caractérisée par l' « universalité » et la «transcendance »; il est le « sommet absolu du développement de la musique universelle, qui mettait un terme au progrès de l'art musical ${ }^{74}$.

En 1857, paraît, toujours en français, un ouvrage sur Beethoven ${ }^{75}$, écrit lui aussi de main de maître, dans une langue riche, avec un art consommé de l'argumentation et de la polémique feutrée, illustré par des analyses où la technique s'allie à une grande sensibilité musicale. Ulybyšev, à la suite de Fétis ou de Berlioz, distinguait trois périodes ${ }^{76}$ dans l'œuvre de Beethoven, la troisième et dernière étant caractérisée par des « dissonances » ou « chimères », et le « reniement des principes élémentaires de l'harmonie ${ }^{77}$, qu'Ulybyšev attribuait en partie à sa surdité. Il s'élevait contre les excès de la «Beethovenomanie », en s'appuyant en particulier sur Fétis. Il sera critiqué par Alexandre Sérov et par Liszt ${ }^{78}$. L. Tolstoj (qui

71. Id., p. 7. Hocquard parle de la culture musicale « prodigieuse » d'Ulybyšev et se dit « stupéfait » de ses « connaissances culturelles » (p. 9,11).

72. Laroš, «O žizni i trudah Ulybyševa » t. 1, p. 1, 2, 3, 6. Les renseignements biographiques donnés par G. Laroš sont tous empruntés à l'article de Gaciskij. Liszt dit du livre d'Ulybyšev que c'était « une œuvre excellente dans son ensemble » (lettre à Wilhelm von Lenz du 2 décembre 1852, in F. Liszt, Correspondance. Lettres choisies, présentées et annotées par Pierre-Antoine Huré et Claude Knepper, P. : JC Lattès, 1987, p. 253).

73. Oulibicheff, Mozart, p. 21.

74. O. Levaševa, Ju. Keldyš, A. Kandinskij, Istorija russkoj muzyki [Histoire de la musique russe], t. 1, M. : Muzyka, 1973,p. 226.

75. Oulibicheff, Beethoven ses critiques et ses glossateurs. Le titre, polémique, vise surtout le musicographe Wilhelm von Lenz (1809-1883), conseiller d'État au ministère de la Justice à Saint-Pétersbourg, auteur de Beethoven et ses trois styles (Saint-Pétersbourg, 1852, écrit (très mal) en français, puis publié en allemand dans une version revue et augmentée), où il critiquait les jugements portés par Ulybyšev dans son Mozart sur le Beethoven de la dernière période. Lenz est aussi l'auteur de souvenirs sur Liszt, Chopin, Tausig et Henselt (Grands virtuoses du piano (1872, dédié aux comtes Michel et Mathieu Wielhorski), traduit de l'allemand et présenté par Jean-Jacques Eigeldinger, Flammarion, 1995).

76. Ulybyšev emploie toujours le mot au masculin, forme maintenant vieillie.

77. Oulibicheff, Beethoven, p. 270

78. Fr. Liszt, « Ulibischeff und Seroff », Neue Zeitschrift für Musik, 48 (1), 1858. F. Fétis conclut ainsi la notice qu'il consacra à Ulybyšev dans sa Biographie universelle des musiciens : «Malheureusement, M. d'Oulibicheff, homme du monde et grand seigneur, n'était pas préparé à ces choses [les critiques] qui sont inséparables de la vie d'artiste et d'écrivain : il en mourut. » (t. 6, p. 391-392). 
avait fait la connaissance d'Ulybyšev chez le compositeur et critique musical Feofil Matveevič Tolstoj le 5 janvier 1857) s'inspire du livre d'Ulybyšev (sans le citer) quand il parle, dans Qu'est-ce que l'art ?, des « ouvrages insensés » de Beethoven et du « délire musical » du compositeur sourd ${ }^{79}$.

Car la musique réalise désormais pour Ulybyšev l'idéal de l'utopie à laquelle il songe encore : elle est cet autre monde « où le code du beau est le seul en vigueur, et dont les habitants, quoique divisés en classes, c'est-à-dire en octaves, sont tous égaux devant la loi harmonique, fraternisent tous dans l'accord et composent ainsi une république heureuse, unie et docile, une république idéale, en un mot, qui transportée dans l'ordre politique serait, sans aucun doute, le meilleur de tous les gouvernements impossibles $»^{80}$.

\section{Université de Caen Basse-Normandie}

m.niqueux@wanadoo.fr

79. L. Tolstö̈, Écrits sur l'art. Rassemblés, présentés et annotés par Lubomir Radoyce, traduit du russe par Maya Minoustchine. P. : Gallimard, 1971, p. 198, 239, 439, 465.

80. Oulibicheff, Beethoven, p. 70. 